\title{
Editorial: Exchanges at the Root-Soil Interface: Resource Trading in the Rhizosphere That Drives Ecosystem Functioning
}

\author{
Catherine Preece $^{1 *}$, Alberto Canarini ${ }^{2}$, Erik Verbruggen ${ }^{1}$ and Lucia Fuchslueger ${ }^{3}$ \\ ${ }^{1}$ Research Group PLECO (Plants and Ecosystems), Department of Biology, University of Antwerp, Antwerp, Belgium, \\ ${ }^{2}$ Center for Ecological Research, Kyoto University, Kyoto, Japan, ${ }^{3}$ Centre of Microbiology and Environmental Systems \\ Science, University of Vienna, Vienna, Austria
}

Keywords: rhizosphere, plant-soil (belowground) feedbacks, volatile organic compound, root exudate, mycorrhiza, soil microorganisms

\section{Editorial on the Research Topic}

Exchanges at the Root-Soil Interface: Resource Trading in the Rhizosphere That Drives Ecosystem Functioning

OPEN ACCESS

Edited and reviewed by: Frank Hagedorn,

Swiss Federal Institute for Forest, Snow and Landscape Research

(WSL), Switzerland

${ }^{*}$ Correspondence:

Catherine Preece

catherine.preece09@gmail.com

Specialty section

This article was submitted to Forest Soils,

a section of the journal Frontiers in Forests and Global

Change

Received: 26 July 2021 Accepted: 05 August 2021 Published: 25 August 2021

Citation:

Preece C, Canarini A, Verbruggen E and Fuchslueger L (2021) Editorial: Exchanges at the Root-Soil Interface: Resource Trading in the Rhizosphere That Drives Ecosystem Functioning. Front. For. Glob. Change 4:747492. doi: 10.3389/ffgc.2021.747492
The interactions between roots, their surrounding soil-the rhizosphere-and soil biota form an intricate web influencing ecosystem functioning. These rhizosphere interactions are not a one-way road but are mostly bi- or multi-directional. For example, roots take up water and nutrients from the soil and deposit carbon (C) used by rhizosphere inhabiting microorganisms for energy and growth. These microorganisms liberate nutrients locked up in soil organic matter, which in turn become available to plants and facilitate plant productivity (Finzi et al., 2015). Hence, tight interactions between plants and soil biota occurring at the rhizosphere scale shape large-scale $\mathrm{C}$ and nutrient cycling and can induce feedbacks at the ecosystem level. The chemical complexity of these interactions goes beyond the mere exchange of carbon and nutrients and can involve a large suite of secondary metabolites, including volatile organic compounds (VOCs). These are produced not only by plants to attract or repel microorganisms and soil fauna, but also by microbes themselves to elicit effects on root growth. Classical examples are associations between roots and mycorrhizal fungi that are initiated by strigolactone exudation of roots (Biate et al., 2015), and nitrogen-fixing bacteria that are attracted by flavonoids produced by their legume hosts (Schulz-Bohm et al., 2018).

Through resolving these crucial processes occurring in the vicinity of roots, research in rhizosphere ecology can advance predictions on the responses of plant productivity, species composition, and soil functioning of forests and other terrestrial ecosystems in a future climate. In this collection we highlight studies investigating rhizosphere resource trading in field and laboratory settings, representing a range of terrestrial ecosystems including temperate, dryland, and tropical climates, in both natural and agro-ecosystems. The complexity of the soil-rhizosphere system is brought into focus through the application of a variety of methods, ranging from root trenching and stable isotope analyses, to fingerprint methods to characterize microbial communities, such as phospholipid fatty acid analysis and DNA amplicon sequencing, to quantitative PCR of functional genes followed up by greenhouse gas (GHG) fluxes.

Associations of roots with mycorrhizal fungi play an essential role in forest soils. Mycorrhizal fungi enlarge the surface area of fine roots and enhance or regulate plant nutrient uptake, allowing 
plants to thrive in nutrient poor soils, emphasizing their role in providing a vital ecosystem service (Smith and Read, 2008). Nevertheless, both tree and mycorrhizal community composition can influence nutrient partitioning in forests. Tatsumi et al. demonstrated that nitrogen $(\mathrm{N})$ uptake of understorey plants growing below different trees in dryland forests in China was linked to their respective mycorrhizal associations. By measuring the natural abundance stable isotopic composition of $\mathrm{N}(\delta 15 \mathrm{~N})$ they found that the identity of overstory trees determined their root mycorrhizal community, and subsequently controlled the $\mathrm{N}$ uptake patterns of understory trees. Mycorrhizal fungi not only transfer nutrients among plants, but also positively or negatively interact with saprotrophic fungi in the breakdown of organic matter (Frey, 2019). In a litter decomposition and root manipulation experiment conducted in a temperate deciduous forest Marañón-Jiménez et al. observed that substrate quality and availability are key factors determining the prevalence of either fungal guild. Ectomycorrhizal fungi were dominant in substrates with low levels of organic matter, whereas saprotrophs were more common at higher levels.

Arbuscular mycorrhizal fungi, in contrast, are considered to play a crucial role for plant phosphorus (P) acquisition (Smith and Smith, 2011). In a tropical lowland forest, Yaffar et al. investigated functional traits, including colonization by mycorrhizal fungi, of fine-roots of trees growing in P-poor soils before and after two consecutive hurricanes. They found that trade-offs between morphological and physiological (phosphatase activity and fungal colonization) root traits related to $\mathrm{P}$ acquisition were largely driven by interspecific differences of pioneer vs. non-pioneer species, and that trait distribution was highly stable to hurricane disturbances. These findings highlight the potential of traits related to mycorrhization and nutrient acquisition, as recently advocated by (Bergmann et al., 2020), to shed light on the community assembly of these diverse forest ecosystems.

Carbon released by plant roots not only affects organisms directly interacting with plant roots, but effects propagate to higher trophic levels through the soil food web. Bluhm et al. indeed found that root inputs are shaping the community composition and abundance of different types of soil fauna in Central European forests. The investigated soil macrofauna groups showed high resilience to deprivation of root resources. In contrast, mesofauna (both decomposers and predators) and root feeding microfauna appeared more sensitive than other groups, highlighting their important role for channeling root derived energy through forest soil food webs.

\section{REFERENCES}

Bergmann, J., Weigelt, A., Van Der Plas, F., Laughlin, D. C., Kuyper, T. W., Guerrero-Ramirez, N., et al. (2020). The fungal collaboration gradient dominates the root economics space in plants. Sci. Adv. 6:eaba3756. doi: $10.1126 /$ sciadv.aba3756

Biate, D. L., Kumari, A., Annapurna, K., Kumar, L. V., Ramadoss, D., Reddy, K. K., et al. (2015). "Legume root exudates: their role in symbiotic interactions," in Plant Microbes Symbiosis: Applied Facets, ed N. K.
Links and interactions between plant and soil microorganisms are bi-directional. In agricultural systems, fertilizer additions can induce strong changes in the composition, functioning and activity of microbial communities (Geisseler and Scow, 2014), which in turn can feed back on crop yield, as well as greenhouse gas emissions. In such a system, Yoshiura et al. tested the application of plant-growth-promoting-rhizobacteria (PGPR) and different fertilizers on maize yield, rhizosphere microbes and soil greenhouse gas emissions. They found that the best option to balance plant biomass production and reduce GHG mitigation in their system was a combination of maize residue retention, which changed rhizosphere communities, and PGPR inoculation to stimulate plant growth via phytohormones. Our knowledge about the array of root inputs to the rhizosphere, in the form of exudates and VOCs produced by rhizosphere microbial communities, continues to expand. Kong et al. investigated VOCs produced by a rhizobacterium which were shown to induce tolerance to iron deficiency stress in Arabidopsis thaliana through changes to a range of root morphological and physiological traits.

This Research Topic highlights the increasing number of studies focusing on exchanges happening at the root-soil interface and their pivotal role in determining numerous ecosystem functions. These exchanges include a multitude of players from plants to soil fauna, and cover a variety of implications from nutrient acquisition to GHGs release. Continuous improvements in experimental manipulations and laboratory analyses are fostering a new understanding of belowground communications and trading, which can translate microscale processes into macroscale ecosystem implications.

\section{AUTHOR CONTRIBUTIONS}

$\mathrm{CP}$ led the writing of the first draft of this Editorial with comments from the other authors. All authors contributed to the article and approved the submitted version.

\section{FUNDING}

CP was funded by an FWO (Research Foundation - Flanders) post-doctoral fellowship. AC received financial support as an International Research Fellow of Japan Society for the Promotion of Science [Postdoctoral Fellowships for Research in Japan (Standard)]. LF acknowledges the European Union's Horizon 2020 research and innovation program under the Marie Sklodovska-Curie grant agreement No 847693 (REWIRE).

Arora (New Delhi: Springer India), 259-271. doi: 10.1007/978-81-322-20 68-8_13

Finzi, A. C., Abramoff, R. Z., Spiller, K. S., Brzostek, E. R., Darby, B. A., Kramer, M. A., et al. (2015). Rhizosphere processes are quantitatively important components of terrestrial carbon and nutrient cycles. Glob. Change Biol. 21, 2082-2094. doi: 10.1111/gcb.12816

Frey, S. D. (2019). Annual review of ecology, evolution, and systematics. Ann. Rev. 50, 237-259. doi: 10.1146/annurev-ecolsys-1106 $17-062331$ 
Geisseler, D., and Scow, K. M. (2014). Long-term effects of mineral fertilizers on soil microorganisms - a review. Soil Biol. Biochem. 75, 54-63. doi: 10.1016/j.soilbio.2014.03.023

Schulz-Bohm, K., Gerards, S., Hundscheid, M., Melenhorst, J., De Boer, W., and Garbeva, P. (2018). Calling from distance: attraction of soil bacteria by plant root volatiles. ISME J. 12, 1252-1262. doi: 10.1038/s41396-017-0035-3

Smith, S. E., and Read, D. J. (2008). Mycorrhizal Symbiosis, 3rd Edn. San Diego and London: Academic Press, 1-769.

Smith, S. E., and Smith, F. A. (2011). Roles of arbuscular mycorrhizas in plant nutrition and growth: new paradigms from cellular to ecosystem scales. Ann. Rev. Plant Biol. 62, 227-250. doi: 10.1146/annurev-arplant-042110-103846

Conflict of Interest: The authors declare that the research was conducted in the absence of any commercial or financial relationships that could be construed as a potential conflict of interest.
Publisher's Note: All claims expressed in this article are solely those of the authors and do not necessarily represent those of their affiliated organizations, or those of the publisher, the editors and the reviewers. Any product that may be evaluated in this article, or claim that may be made by its manufacturer, is not guaranteed or endorsed by the publisher.

Copyright (c) 2021 Preece, Canarini, Verbruggen and Fuchslueger. This is an open-access article distributed under the terms of the Creative Commons Attribution License (CC BY). The use, distribution or reproduction in other forums is permitted, provided the original author(s) and the copyright owner(s) are credited and that the original publication in this journal is cited, in accordance with accepted academic practice. No use, distribution or reproduction is permitted which does not comply with these terms. 